\title{
Zero-field magnetometry using hyperfine-biased nitrogen-vacancy centers near diamond surfaces
}

\author{
Ning Wang $\odot,{ }^{1,2,3}$ Chu-Feng Liu, ${ }^{1}$ Jing-Wei Fan $\odot,{ }^{1}$ Xi Feng, ${ }^{1}$ Weng-Hang Leong, ${ }^{1}$ Amit Finkler $\odot,{ }^{4,5,}{ }^{*}$ Andrej Denisenko, ${ }^{4,5}$ \\ Jörg Wrachtrup, ${ }^{4,5}$ Quan Li®,$^{1,2,3, \dagger}$ and Ren-Bao Liu $\odot^{1,2,3, \ldots}$ \\ ${ }^{1}$ Department of Physics, The Chinese University of Hong Kong, Shatin, New Territories, Hong Kong, China \\ ${ }^{2}$ Centre for Quantum Coherence, The Chinese University of Hong Kong, Shatin, New Territories, Hong Kong, China \\ ${ }^{3}$ The Hong Kong Institute of Quantum Information Science and Technology, The Chinese University of Hong Kong, \\ Shatin, New Territories, Hong Kong, China \\ ${ }^{4} 3 r d$ Institute of Physics and Center for Applied Quantum Technologies, University of Stuttgart, 70569 Stuttgart, Germany \\ ${ }^{5}$ Max Planck Institute for Solid State Research, 70569 Stuttgart, Germany
}

(Received 8 September 2021; accepted 14 January 2022; published 9 February 2022)

\begin{abstract}
Shallow nitrogen-vacancy (NV) centers in diamond are promising for nanomagnetometry, for they can be placed proximate to targets. To study the intrinsic magnetic properties, zero-field magnetometry is desirable. However, for shallow NV centers under zero field, the strain near diamond surfaces would cause level anticrossing between the spin states, leading to clock transitions whose frequencies are insensitive to magnetic signals. Furthermore, the charge noises from the surfaces would induce extra spin decoherence and hence reduce the magnetic sensitivity. Here, we demonstrate that the relatively strong hyperfine coupling $(130 \mathrm{MHz})$ from a first-shell ${ }^{13} \mathrm{C}$ nuclear spin can provide an effective bias field to an $\mathrm{NV}$ center spin so that the clock-transition condition is broken and the charge noises are suppressed. The hyperfine bias enhances the dc magnetic sensitivity by a factor of 22 in our setup. With the charge noises suppressed by the strong hyperfine field, the ac magnetometry under zero field also reaches the limit set by decoherence due to the nuclear spin bath. In addition, the $130 \mathrm{MHz}$ splitting of the NV center spin transitions allows relaxometry of magnetic noises simultaneously at two well-separated frequencies $(\sim 2.870 \pm 0.065 \mathrm{GHz})$, providing (low-resolution) spectral information of high-frequency noises under zero field. The hyperfine-bias-enhanced zero-field magnetometry can be combined with dynamical decoupling to enhance single-molecule magnetic resonance spectroscopy and to improve the frequency resolution in nanoscale magnetic resonance imaging.
\end{abstract}

DOI: 10.1103/PhysRevResearch.4.013098

\section{INTRODUCTION}

Nitrogen-vacancy (NV) centers [1-3] in diamond are promising quantum sensors of magnetic field [4-6], electric field [7], temperature [8-11], pressure [12], deformation [13], etc., for their long coherence time and correspondingly sharp spectral lines [14]. Shallow NV centers in diamond are suitable for studying nanomagnetism, such as vortices in superconductors $[15,16]$, skyrmions in magnetic thin films $[17,18]$, spin-wave excitations in magnetic insulators [19-21], antiferromagnetism in thin films [22,23], and ferromagnetism of two-dimensional (2D) van der Waals crystals [24]. However, the sensitivity of shallow NV centers is greatly impaired by local strains and electrical noises from surfaces [25-28], which are particularly serious under zero field, a condition often needed for measuring the intrinsic magnetic properties

\footnotetext{
* Present address: Department of Chemical and Biological Physics, Weizmann Institute of Science, Rehovot, Israel.

†liquan@phy.cuhk.edu.hk

‡rbliu@cuhk.edu.hk

Published by the American Physical Society under the terms of the Creative Commons Attribution 4.0 International license. Further distribution of this work must maintain attribution to the author(s) and the published article's title, journal citation, and DOI.
}

of materials [29-33]. Under zero field, the strain causes level anticrossing (LAC) of the spin states, leading to clock transitions whose frequencies are invariant in the first order of magnetic field and therefore are insensitive to weak magnetic fields $[34,35]$. Furthermore, the symmetry breaking by the strain makes the spin transition frequency sensitive to electrical noises, which significantly reduces the spin coherence times of shallow NV centers [36]. A biasing magnetic field can shift the spin transitions away from the clock transition to suppress the effects of strain and electrical noises, but when applied to studying magnetism, it would inevitably perturb the intrinsic properties of the targets. For example, the critical fluctuations of $2 \mathrm{D}$ magnets are suppressed by a merely $30 \mu \mathrm{T}$ field [31], and the magnetic textures such as skyrmions in ultrathin magnetic films are sensitive to magnetic fields as weak as tens of microteslas [32,33].

Here, we propose and demonstrate that the relatively strong hyperfine coupling to a nearby ${ }^{13} \mathrm{C}$ nuclear spin [Fig. 1(a)] can effectively bias an NV center spin for enhancing zero-field magnetometry. The hyperfine coupling to a first-shell ${ }^{13} \mathrm{C}$ $(\sim 130 \mathrm{MHz})$ amounts to a magnetic field on the NV center electron spin $\sim \pm 2.3 \mathrm{mT}$ for the up/down state of the ${ }^{13} \mathrm{C} \mathrm{nu}-$ clear spin. Considering that the strain effect on a shallow NV center spin is usually $<10 \mathrm{MHz}$, such a hyperfine coupling can shift the spin resonances far away from the LAC, recovering the sensitivity of the spin resonance frequencies to weak 
(a)

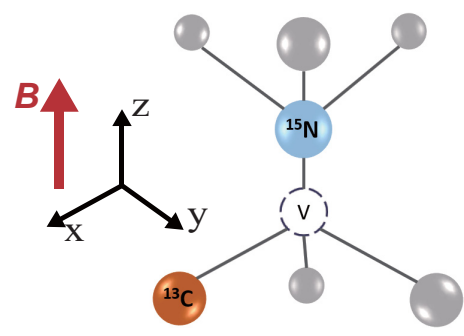

(c)

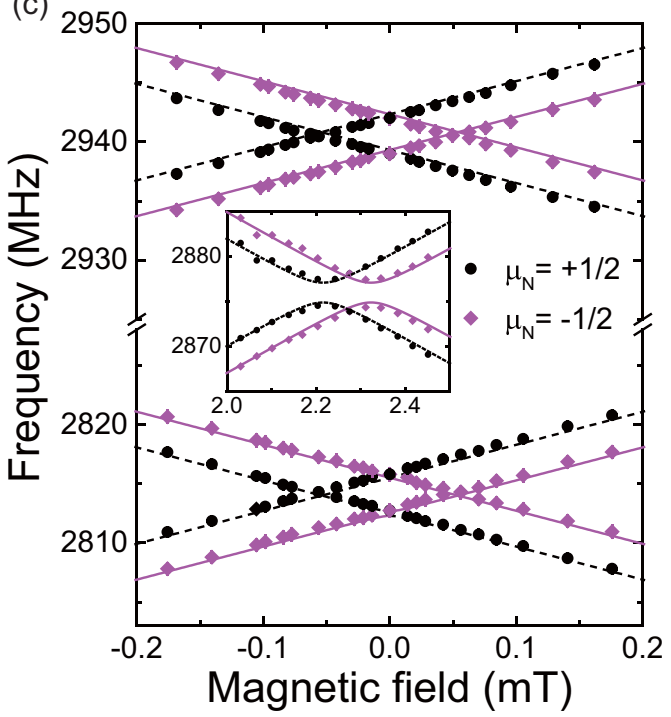

(b)
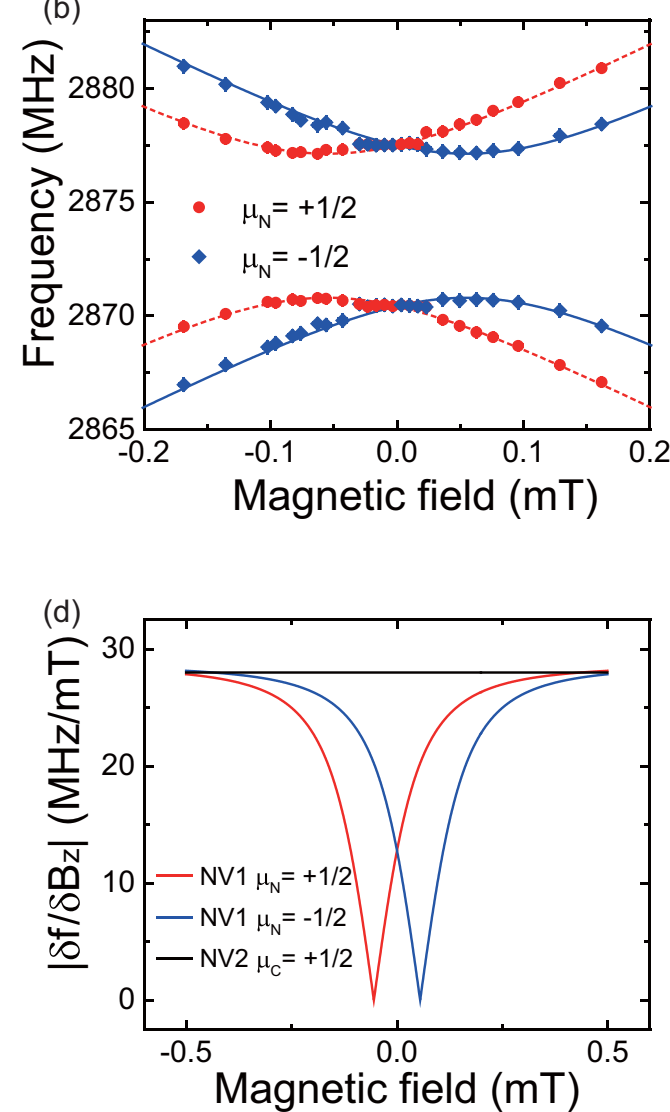

FIG. 1. Hyperfine spin resonances of nitrogen-vacancy (NV) centers. (a) Structure of an NV center with a host ${ }^{15} \mathrm{~N}$ nuclear spin and a first-shell ${ }^{13} \mathrm{C}$ nuclear spin. The external magnetic field $\mathbf{B}$ is applied along the NV axis (the $z$ axis), and the $x$ axis is defined by the strain. (b) Spin resonance frequencies of an NV center without a strongly coupled ${ }^{13} \mathrm{C}(\mathrm{NV} 1)$ as functions of the magnetic field. The red dots and blue diamonds are for the ${ }^{15} \mathrm{~N}$ nuclear spin states with $\mu_{\mathrm{N}}=+\frac{1}{2}$ and $-\frac{1}{2}$, respectively. The corresponding lines are calculated results with strains $\Pi_{x}=3.16 \mathrm{MHz}$ and $\Pi_{z}=3.96 \mathrm{MHz}$. (c) Spin resonance frequencies of an NV center with a first-shell ${ }^{13} \mathrm{C}(\mathrm{NV} 2-\mathrm{C} 13)$. The black dots and purple diamonds are for the ${ }^{15} \mathrm{~N}$ nuclear spin states $\mu_{\mathrm{N}}=+\frac{1}{2}$ and $-\frac{1}{2}$, respectively. The inset shows results at fields close to the level anticrossing $(\mathrm{LAC})$ at $B_{z} \approx 2.3 \mathrm{mT}$. The corresponding lines are calculated results with $\Pi_{x}=2.20 \mathrm{MHz}$ and $\Pi_{z}=1.10 \mathrm{MHz}$. (d) Susceptibilities of spin resonance frequencies to the magnetic field $\left|\partial f / \partial B_{z}\right|$ near zero magnetic field for NV1 (red and blue) and NV2-C13 (black).

magnetic signals and simultaneously suppressing the effects of electrical noises. The biasing field from the nuclear spin has no effect on sensing targets since it decays rapidly $\left(\sim r^{-3}\right)$ with the distance $r$. An additional advantage of the hyperfinebiased NV magnetometry is that the relaxometry (i.e., the measurement of the spin relaxation rate $T_{1}^{-1}$, which equals the noise power density at the spin transition frequency) can be simultaneously carried out at two well-separated frequencies $(\sim 2.870 \pm 0.065 \mathrm{GHz})$ even under zero external field. We use an NV center coupled to a first-shell ${ }^{13} \mathrm{C}$ to measure magnetization of single magnetic nanoparticles (MNPs) and demonstrate that the multifrequency relaxometry can extract coarse-grained spectral information of high-frequency $(\sim 2.8 \mathrm{GHz})$ magnetic fluctuations under zero field.

\section{ZERO-FIELD MAGNETOMETRY ENHANCED BY HYPERFINE BIAS}

The transition frequencies of the NV center electron spin $(S=1)$ were measured by optically detected magnetic resonance (ODMR), in which the spin was initialized by optical excitation, manipulated by microwave pulses, and read out through spin-dependent photoluminescence (for details see Sec. V) [1]. We fabricated nanopillar arrays on a diamond membrane to increase the photon collection efficiency. One to a few shallow NV centers were created in each pillar by ${ }^{15} \mathrm{~N}$ ion implantation with a dose of 100 ions $/ \mu \mathrm{m}^{2}$ at energy of $10 \mathrm{keV}$ [37].

The Hamiltonian of the NV center electron spin and the ${ }^{13} \mathrm{C}$ spin is

$$
\begin{aligned}
H= & \left(D_{\mathrm{gs}}+\Pi_{z}\right) S_{z}^{2}+\Pi_{x}\left(S_{y}^{2}-S_{x}^{2}\right)+\gamma_{\mathrm{e}} B_{z} S_{z}+S_{z} \mathbf{A}_{\mathrm{N}} \cdot \mathbf{I}_{\mathrm{N}} \\
& +S_{z} \mathbf{A}_{\mathrm{C}} \cdot \mathbf{I}_{\mathrm{C}},
\end{aligned}
$$

where $D_{\mathrm{gs}}=2.87 \mathrm{GH} z$ is the ground state zero-field splitting, $\Pi_{x / y / z}$ is the strain along the $x / y / z$ axis, $\mathbf{S}$ is the electronic spin, $\mathbf{I}_{\mathrm{N}}$ is the ${ }^{15} \mathrm{~N}$ nuclear spin- $\frac{1}{2}, \mathbf{I}_{\mathrm{C}}$ is the ${ }^{13} \mathrm{C}$ nuclear spin- $\frac{1}{2}$, $\gamma_{\mathrm{e}}$ is the gyromagnetic ratio of the electron spin, $B_{z}$ is the $z$ component of an external magnetic field, and $\mathbf{A}_{\mathrm{N}}$ and $\mathbf{A}_{\mathrm{C}}$ are the hyperfine coupling vectors for the nitrogen and ${ }^{13} \mathrm{C}$ spins when the NV center spin is along the $z$ axis. In our experiment, we kept the magnetic field along the NV axis as 
much as possible. Though a transverse field $<0.1 \mathrm{mT}$ cannot be fully avoided, it had little effect on our experiments. Details on the alignment of the magnetic field are given in Sec. V. Considering that the transverse magnetic field and hyperfine couplings are weak as compared with the zero-field splitting $D_{\text {gs }}$, we have dropped the nonsecular terms, i.e., those linear in $S_{x}$ and $S_{y}$. We have set the $x y$ coordinates such that $\Pi_{y}=0$. Under the weak magnetic field condition, the Zeeman energies of the nuclear spins are neglected.

The eigenenergies of the system in Eq. (1) are (details in Appendix A)

$$
f_{m_{s}, \mu_{\mathrm{N}}, \mu_{\mathrm{C}}}=D_{\mathrm{gs}}+\Pi_{z}+m_{s} \sqrt{\left(\gamma_{\mathrm{e}} B_{z}+\mu_{\mathrm{N}} A_{\mathrm{N}}+\mu_{\mathrm{C}} A_{\mathrm{C}}\right)^{2}+\Pi_{x}^{2}},
$$

where $m_{s}=0, \pm 1$ labels different electron spin states, and $\mu_{\mathrm{N}}= \pm \frac{1}{2}$ and $\mu_{\mathrm{C}}= \pm \frac{1}{2}$ label the respective nuclear spin states. The hyperfine coupling to the host ${ }^{15} \mathrm{~N}$ is $A_{\mathrm{N}}=3.15$ $\mathrm{MHz}$ [38]. When there is no strongly coupled ${ }^{13} \mathrm{C}$ spin $\left(A_{\mathrm{C}}=0\right)$ and the magnetic field is small $\left(\left|\gamma_{\mathrm{e}} B_{z} \pm A_{\mathrm{N}} / 2\right| \ll\right.$ $\Pi_{x}$ ), the spin resonance frequencies depend quadratically on the magnetic field due to the $\operatorname{LAC}[34,35]$. When there is a ${ }^{13} \mathrm{C}$ nuclear spin located in the first shell $\left(A_{\mathrm{C}} \approx 130 \mathrm{MHz} \gg \Pi_{x}\right)$ [39], the eigenenergies near zero field are

$$
f_{m_{s}, \mu_{\mathrm{N}}, \mu_{\mathrm{C}}} \approx D_{\mathrm{gs}}+\Pi_{z}+m_{s}\left(\gamma_{\mathrm{e}} B_{z}+\mu_{\mathrm{N}} A_{\mathrm{N}}+\mu_{\mathrm{C}} A_{\mathrm{C}}\right),
$$

depending linearly on the external field $B_{z}$.

For comparison, we chose two NV centers with the same crystallographic direction in two different pillars, one with no ${ }^{13} \mathrm{C}$ spin nearby (NV1) and one with a first-shell ${ }^{13} \mathrm{C}$ (NV2-C13) [Fig. 1(a)]. Figure 1(b) shows the ODMR frequencies of NV1 as functions of magnetic field (see Fig. 6 in $\mathrm{Sec} . \mathrm{V}$ for the spectra). The resonance frequencies present two branches with the LAC at $B_{z}=\mu_{\mathrm{N}} \mathrm{A}_{\mathrm{N}} / \gamma_{\mathrm{e}} \approx \mp 0.05 \mathrm{mT}$ corresponding to the ${ }^{15} \mathrm{~N}$ spin state $\mu_{\mathrm{N}}= \pm \frac{1}{2}$. The data are well reproduced by Eq. (2) with fitting parameters $\Pi_{x}=3.16 \mathrm{MHz}$ and $\Pi_{z}=3.96 \mathrm{MHz}$. Due to the strain, the spin resonance frequencies are insensitive to the magnetic field in the range $\left|B_{z} \pm A_{\mathrm{N}} / 2\right|<\Pi_{x}$. The strong hyperfine coupling in NV2C13 leads to two well-separated sets of resonances $\sim 2815$ and $2940 \mathrm{MHz}$ near zero field $(B<0.2 \mathrm{mT})$ [Fig. 1(c)], which depend linearly on the magnetic field. The LACs are shifted to $B_{z}=\mu_{\mathrm{C}} A_{\mathrm{C}} / \gamma_{\mathrm{e}} \approx \mp 2.25 \mathrm{mT}$ for the two eigenstates of the ${ }^{13} \mathrm{C}$ spin [inset in Fig. 1(c)]. The experimental data of NV2-C13 are best fitted with strains $\Pi_{x}=2.20 \mathrm{MHz}$ and $\Pi_{z}=1.10 \mathrm{MHz}$. The susceptibilities $\left(\left|\partial f / \partial B_{z}\right|\right)$ of the two NV centers near zero field [Fig. 1(d)], derived from Eq. (2) with the strain parameters obtained from Figs. 1(b) and 1(c), are largely suppressed $\left(\ll \gamma_{\mathrm{e}}\right)$ by the strain in NV1 but are recovered to $\gamma_{\mathrm{e}}$ by the hyperfine bias of the first-shell ${ }^{13} \mathrm{C}$ in NV2-C13.

The sensitivity of magnetometry also depends on the spin coherence time. We measured the spin coherence time $T_{2}^{*}$ using Ramsey interference (see Fig. 7 in Appendix B for examples). For NV1, the coherence time $T_{2}^{*}$ drops significantly at the LAC [Fig. 2(a)]. Since at the LAC the resonance frequencies are insensitive to the variation of the magnetic field, the dephasing due to magnetic noises should have been suppressed [34-36]. Therefore, the coherence time dropping at the LAC indicates that the increased decoherence of NV1 at the LAC is mainly caused by the enhancement of the effects of electrical noises by the strain, which is consistent with literature [25-28,36] and simulation (see Appendix B). For NV2-C13, the strong hyperfine interaction shifts the LAC away from the zero field, so the coherence time is nearly a constant near zero field [Fig. 2(b)]. The coherence time of NV2-C13 near zero field is longer than near its LAC [inset of Fig. 2(b)] since the strong hyperfine bias suppresses the effects of electrical noises.

The dc magnetic sensitivity by Ramsey measurement depends on the susceptibility and the spin coherence time by [40]

$$
\eta \approx\left|\frac{\partial f}{\partial B_{z}}\right|^{-1} \frac{\exp \left[\left(\tau / T_{2}^{*}\right)^{2}\right]}{C \sqrt{P_{0} t_{\mathrm{R}}}} \frac{\sqrt{t_{I}+\tau+t_{R}}}{\tau},
$$

where $t_{\mathrm{I} / \mathrm{R}}$ is the spin initialization/readout time, $C$ is the signal contrast of the Ramsey interference, and $P_{0}$ is the photon count rate. In our experiments, $t_{\mathrm{I}}=t_{\mathrm{R}}=300 \mathrm{~ns}$ and $T_{2}^{*}$ is several microseconds. The optimal sensitivity is taken at $\tau=T_{2}^{*} / 2$ [40]. For NV1, the saturated photon count rate $P_{0} \approx 360 \mathrm{kcps}$ and $C \approx 0.11$; for NV2-C13, $P_{0} \approx 440 \mathrm{kcps}$ and $C \approx 0.11$. As shown in Fig. 2(c), the magnetic sensitivity of NV1 near zero field is $\sim 20$ times worse than that under a bias magnetic field. The sensitivity of NV2-C13 is nearly invariant in the small magnetic regime and is significantly better than that of NV1, owing to its longer coherence time $T_{2}^{*}$ and large susceptibility $\left|\partial f / \partial B_{z}\right|$.

We demonstrate the dc sensitivities of the NV sensors using real-time magnetic field measurement. We used an electromagnet to generate small magnetic field variations. The sensitivity of the pulsed ODMR approaches that of the Ramsey sequence in Eq. (4) [40]. For fast measurement, we adopted the two-point method, in which the photon counts $C\left(f_{i}\right)$ were recorded in pulsed ODMR at two microwave frequencies [41], one $\left(f_{1}\right)$ at the half maximum of the ODMR resonance and the other $\left(f_{2}\right)$ far away from the resonance [inset of Fig. 2(d)]. The signal $S=\frac{C\left(f_{2}\right)-C\left(f_{1}\right)}{C\left(f_{2}\right)}$ was measured as a function of time. The dependence of the signal on the field change $\delta B$ is $\delta S=\frac{C^{\prime}\left(f_{1}\right)}{C\left(f_{2}\right)} \frac{\partial f}{\partial B_{z}} \delta B_{z}$, where $C^{\prime} \equiv \frac{\partial C}{\partial f}$. Due to the strain effect, $\left|\frac{\partial f}{\partial B_{z}}\right| \ll \gamma_{\mathrm{e}}$ for NV1 near zero field. When a biased magnetic field $\sim 0.5 \mathrm{mT}$ is applied, $\left|\frac{\partial f}{\partial B_{z}}\right|=\gamma_{\mathrm{e}}$. As shown in Fig. 2(d), NV1 cannot detect the variation between 14 and $26 \mu \mathrm{T}$ but can detect both the variations from 516 and 528 to $520 \mu \mathrm{T}$. In contrast, NV2-C13 can detect the small field variations with or without the bias field, with almost invariant signal-to-noise ratio.

As for the measurement of an ac magnetic field, the sensitivity can be improved by removing the effect of the static or slow-varying noises using spin echo or dynamical decoupling $[6,42]$. To evaluate the sensitivity of ac magnetometry, we measured the spin echo coherence time $T_{2}$ of the NV sensors near zero field. The ac magnetic sensitivity, for measurement with spin echo, is $\eta_{\mathrm{ac}} \sim \eta_{\mathrm{dc}} \sqrt{T_{2}^{*} / T_{2}}$. The coherence time $T_{2}$ of NV1 [Fig. 3(a)] drops dramatically near zero field, being shorter by almost one order of magnitude than that under a bias field of $0.1 \mathrm{mT}$. The $T_{2}$ time of NV1 is partially recovered at the clock transition points $B_{z}=\mp \frac{A_{N}}{2 \gamma_{\mathrm{e}}}$ due to the suppression 

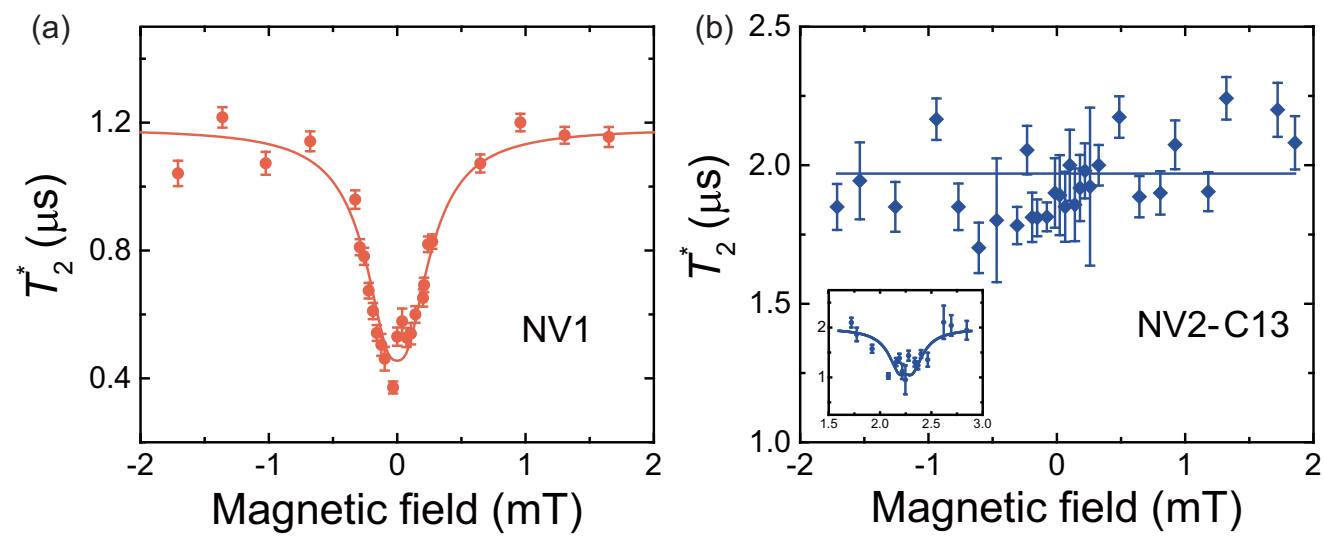

(c)

(d)
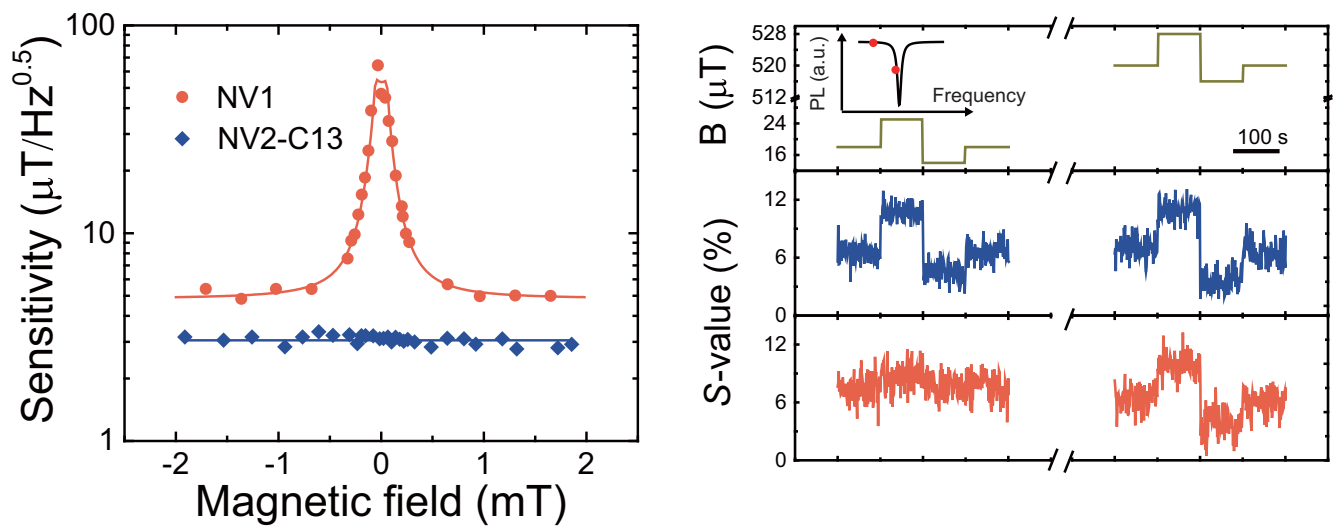

FIG. 2. Zero-field dc magnetometry by a nitrogen-vacancy (NV) center without or with a first-shell ${ }^{13} \mathrm{C}$. (a) and (b) Spin coherence time $\left(T_{2}^{*}\right)$ of NV1 and NV2-C13, respectively. The dots are measured data, and the lines are fitting curves. Inset in (b) shows results near the level anticrossing (LAC). (c) Sensitivity of NV1 (orange) and NV2-C13 (blue), estimated with Eq. (4) using the parameters extracted from the experimental data. (d) Real-time magnetic field measurement using NV1 and NV2-C13. The two microwave frequencies $f_{1 / 2}$ are indicated by red dots in the inset of the upper panel. The acquisition time is $0.2 \mathrm{~s}$ per frequency. An electromagnet is used to generate magnetic field as shown in the upper panel. The middle and lower panels show the measured results by NV2-C13 and NV1, respectively.

of the magnetic noises by the LAC. The overall drop of coherence time near zero field and the partial recovery of coherence time at the LAC suggest that both electrical and magnetic noises contribute to the echo decoherence, and the former is stronger. The field dependence of the spin coherence time of NV2-C13 is in good agreement with the nuclear spin bath theory [43], which confirms that the electrical noises are largely suppressed by the hyperfine bias. In the medium-weak field regime $\left(0.1 \mathrm{mT}<B_{z} \ll 30 \mathrm{mT}\right)$, the decoherence is mainly caused by the nuclear spin precession under the magnetic field and the hyperfine field (with the latter depending on the central spin state), and the coherence time increases (a)

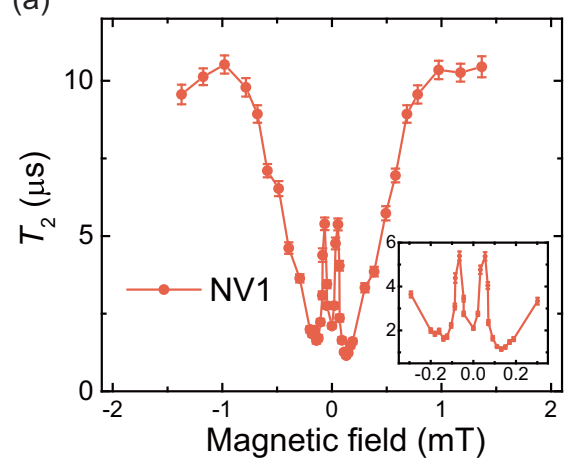

(b)

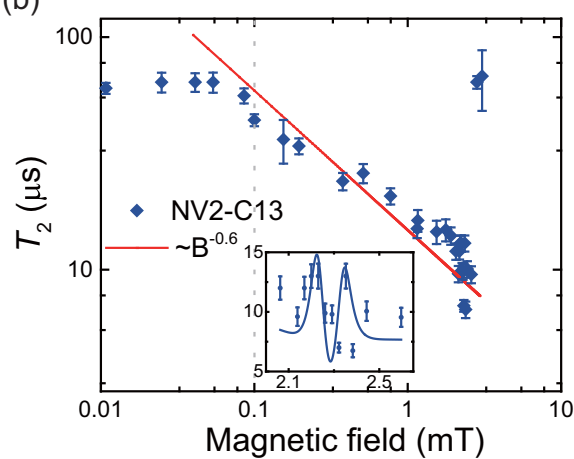

(c)

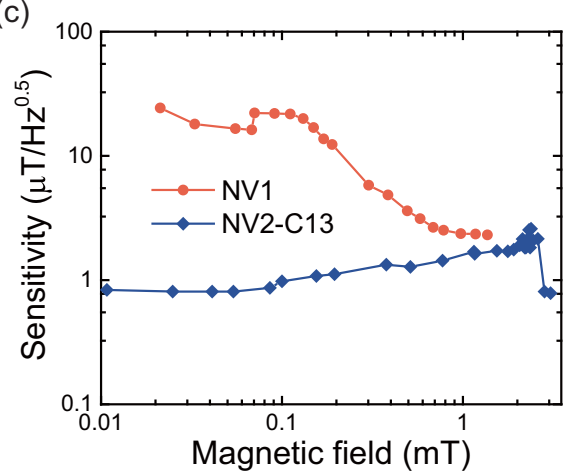

FIG. 3. Echo coherence time and sensitivity of ac magnetometry by a nitrogen-vacancy (NV) center without or with a first-shell ${ }^{13} \mathrm{C}$. (a) and (b) Spin coherence time $\left(T_{2}\right)$ of NV1 and NV2-C13, respectively. Inset in (a) zooms in the field dependence near the level anticrossing (LAC). (c) Sensitivities of NV1 (orange) and NV2-C13 (blue) using the parameters extracted from the experimental data as functions of magnetic field. 

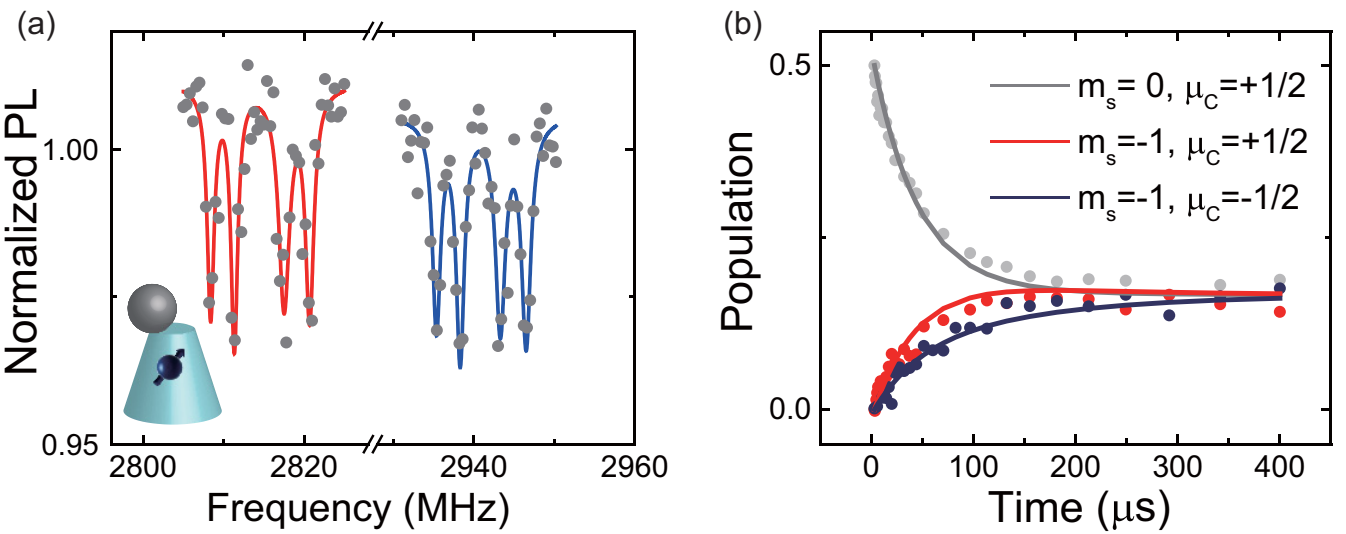

FIG. 4. Two-frequency zero-field relaxometry enabled by hyperfine coupling to a first-shell ${ }^{13} \mathrm{C}$ nuclear spin. (a) Optically detected magnetic resonance (ODMR) spectrum of NV3-C13 with a magnetic nanoparticle placed in the proximity. (b) Populations in the states $\left|m_{s}=-1, \mu_{\mathrm{C}}=+\frac{1}{2}\right\rangle$ (red), $\left|m_{s}=-1, \mu_{\mathrm{C}}=-\frac{1}{2}\right\rangle$ (navy), and $\left|m_{s}=0, \mu_{\mathrm{C}}=+\frac{1}{2}\right\rangle$ (gray) as functions of time after the initialization.

with decreasing the magnetic field with dependence $\propto B_{z}^{-0.6}$ [43]. When the magnetic field is weaker than $0.1 \mathrm{mT}$, the nuclear spin precession frequencies would be mostly determined by the hyperfine couplings, so the coherence time saturates. These results indicate that the strong hyperfine bias can push the ac magnetometry sensitivity to the limit set by nuclear spin-induced decoherence. As shown in Fig. 3(c), the ac sensitivity of NV1 is much worse than that of NV2-C13 in the low-field regime $(<0.1 \mathrm{mT})$. With increasing field $(>1 \mathrm{mT})$, the sensitivities of NV1 and NV2-C13 become comparable. Near the LAC of NV2-C13 $\left(B_{z} \approx \pm 2.3 \mathrm{mT}\right)$, the sensitivity of NV2-C13 is also reduced by the strain effect.

\section{MULTIPLE-FREQUENCY RELAXOMETRY NEAR ZERO FIELD}

Another advantage of the strong hyperfine bias comes from the well-separated electron spin resonances under zero field. These resonances can be used for zero-field relaxometry at multiple frequencies, offering partial, although very coarsegrained, spectral information of high-frequency noises. In relaxometry, the spin relaxation time $T_{1}$ between two states with a transition frequency $f_{i}$ is related to the noise power spectrum at the frequency $S\left(2 \pi f_{i}\right)$ by $[2,3,40]$

$$
T_{1}^{-1}=\gamma_{\mathrm{e}}^{2} S\left(2 \pi f_{i}\right) \text {. }
$$

Under zero/low magnetic fields, the conventional NV relaxometry can only detect the noise at one frequency $(\sim 2.87$ $\mathrm{GHz}$ ). With the NV spin resonance split by the $130 \mathrm{MHz}$ coupling of a first-shell ${ }^{13} \mathrm{C}$ nuclear spin, the noise intensities at two frequencies $(\sim 2.870 \pm 0.065 \mathrm{GHz})$ can be measured under zero field. The multifrequency relaxometry, though with a low resolution, can provide valuable information for understanding the intrinsic dynamics of the MNP (without the interference from an external field).

To demonstrate the zero-field two-frequency relaxometry, we used a shallow NV center coupled to a first-shell ${ }^{13} \mathrm{C}$ (NV3-C13) to measure the magnetic noise from an MNP. The spin relaxation rate of this NV center without the MNP is $0.15(1) \mathrm{ms}^{-1}$, approaching the typical values in bulk diamond. We dropcasted the ethanol solution containing ferromagnetic copper-nickel MNPs (100-300 $\mathrm{nm}$ in diameter) on the diamond surface [41]. The presence of a nearby MNP is evidenced by the splitting of the NV resonances [Fig. 4(a)]. The spin relaxation times are significantly shorter than before the introduction of the MNP. The different relaxation dynamics of the states $\left|m_{s}=0, \mu_{\mathrm{C}}=-\frac{1}{2}\right\rangle, \mid m_{s}=-1, \mu_{\mathrm{C}}=$ $\left.+\frac{1}{2}\right\rangle$, and $\left|m_{s}=-1, \mu_{\mathrm{C}}=-\frac{1}{2}\right\rangle$ [Fig. 4(b)] result from the unequal relaxation rates between the states. Solving the rate equation (details in Appendix $\mathrm{C}$ ), we obtain the transition rate between $\left|m_{s}=0, \mu_{\mathrm{C}}=-\frac{1}{2}\right\rangle$ and $\left|m_{s}=-1, \mu_{\mathrm{C}}=-\frac{1}{2}\right\rangle$ is $5.0(4) \mathrm{ms}^{-1}$, while that between $\left|m_{s}=0, \mu_{\mathrm{C}}=+\frac{1}{2}\right\rangle$ and $\left|m_{s}=-1, \mu_{\mathrm{C}}=+\frac{1}{2}\right\rangle$ is $8.9(3) \mathrm{ms}^{-1}$, larger than the former one by $78 \%$. Similarly, the transition rate between $\mid m_{s}=0$, $\left.\mu_{\mathrm{C}}=+\frac{1}{2}\right\rangle$ and $\left|m_{s}=+1, \mu_{\mathrm{C}}=+\frac{1}{2}\right\rangle$ is 6.0(4) $\mathrm{ms}^{-1}$, while that between $\left|m_{s}=0, \mu_{C}=-\frac{1}{2}\right\rangle$ and $\left|m_{s}=+1, \mu_{C}=-\frac{1}{2}\right\rangle$ is $10.0(5) \mathrm{ms}^{-1}$, larger than the former by $67 \%$. These differences suggest that the noise from the MNP has a subgigahertz spectral width. The observations suggest that both Johnson noise and spin-wave excitation contribute to the decay of spin lifetime $[19,44,45]$. As the MNP is metallic with good conductivity, Johnson noises can result from thermal current fluctuations, which interact with the NV center spin, inducing a relaxation rate $[44,45] \Gamma_{1}=T_{1}^{-1} \propto \frac{k_{\mathrm{B}} T \sigma}{z_{\mathrm{NV}}^{2}}$, where $\sigma$ is the conductivity of the material, and $z_{\mathrm{NV}}$ is the sample-to-probe distance. The relaxation rate $\left(5-9 \mathrm{~ms}^{-1}\right)$ is increased by nearly one order of magnitude from that without coupling to the MNP $\left(0.15 \mathrm{~ms}^{-1}\right)$, which is consistent with the results in the literature for similar conductivity and sample-to-probe distance [44]. However, as indicated by the two-frequency relaxometry, the relaxation rate of the transition with higher frequency $\left(\sim 9 \mathrm{~ms}^{-1}\right)$ is smaller than that with lower frequency $\left(\sim 5 \mathrm{~ms}^{-1}\right)$. Since the Johnson noise is a white noise, such frequency dependence suggests an additional color noise, which we attribute to the magnetic noise due to, e.g., spin-wave excitations. As the difference of the two frequencies is $\sim 130 \mathrm{MHz}$, the magnetic noise should have a spectral width in the order of hundreds of megahertz, which is consistent with the observation in Ref. [19]. However, more experiments are needed for definite designation of the noise sources. 


\section{CONCLUSIONS AND DISCUSSION}

To conclude, we have proposed a scheme to utilize strongly coupled ${ }^{13} \mathrm{C}$ nuclear spins to bias the NV center spin resonances, which can suppress the effect of electrical noises from surfaces, and to remove the insensitivity of the resonance frequencies near the LAC due to strain under zero or very weak field. The sensitivity of both dc and ac magnetometry under zero field is significantly improved by the strong hyperfine bias. It should be mentioned that, exactly at the level-crossing points, the two degenerate transitions $(|0\rangle \leftrightarrow| \pm 1\rangle)$ shift oppositely under a weak magnetic signal, which results in insensitivity to a magnetic signal if the transitions cannot be selected. Selective excitation of a magnetically sensitive transition $(|0\rangle \leftrightarrow|+1\rangle$ or $|0\rangle \leftrightarrow|-1\rangle)$ at exact level-crossing has been realized using circularly polarized microwave pulses $[46,47]$. Nuclear spins were also proposed to remove the exact degeneracy at zero field in Ref. [47] (but not to overcome the strain effect). The Raman dynamical decoupling schemes in Refs. $[48,49]$, which use linearly polarized microwave pulses, can be adopted to enhance ac magnetometry near the levelcrossing points.

The strong coupling to the nuclear spin enables relaxometry at well-separated frequencies under weak field, which can provide partial spectral information of the noise. It is possible to have $>1$ strongly coupled ${ }^{13} \mathrm{C}$ spins. Multiple ${ }^{13} \mathrm{C}$ spins (or some other dark spins such as P1 center spins) can enable noise spectroscopy at more frequencies (e.g., $2.87 \pm 0.065$ and $2.87 \pm 0.130 \mathrm{GHz}$ for two ${ }^{13} \mathrm{C}$ spins in the first shell). The hyperfine-biased NV magnetometry near zero field will be useful for the study of critical magnetic fluctuations of nanosystems, magnetic dynamics of soft magnetic materials, magnetic resonance imaging of delicate textures, all of which require minimum disturbance from external magnetic field.

In our experiments, the probability of finding an $\mathrm{NV}$ center coupled to a first-shell ${ }^{13} \mathrm{C}$ nuclear spin is $\sim 3 \%$ in naturalabundance diamond samples (with $1.1 \%{ }^{13} \mathrm{C}$ isotope), which is not extremely rare. In addition, ${ }^{13} \mathrm{C}$ nuclear spins located at adjacent shells can also be used if the hyperfine coupling is larger than the strength of strains $\left(A_{C}>\Pi_{\perp}\right)$. For example, for strains $\sim 5 \mathrm{MHz}$, the possibility for finding an $\mathrm{NV}$ center with $A_{C}>6 \mathrm{MHz}$ is $\sim 28.9 \%$ [50]. The probability of finding an $\mathrm{NV}$ center coupled to two first-shell ${ }^{13} \mathrm{C}$ nuclear spin is $\sim 0.03 \%$, and that of finding an NV center coupled to two ${ }^{13} \mathrm{C}$ spins with $A_{C}>6 \mathrm{MHz} \sim 4.1 \%$. Isotope enrichment [51,52] can increase the probability for finding NV centers strongly coupled to ${ }^{13} \mathrm{C}$ spins. The enrichment may deteriorate the coherence to some extent. However, as the main decoherence factor for shallow NV centers is the noises from the surfaces, the isotope enrichment would only slightly reduce $T_{2}^{*}$, with minor effects on dc magnetometry. In ac magnetometry, dynamical decoupling can be applied to suppress the noise from nuclear spins even at high isotope enrichment, which can prolong the coherence time up to $T_{1}$ [43].

\section{METHODS}

The ODMR setup was built on a commercial microscope frame. The continuous-wave laser beam from a solid-state laser (MGL-III-532-200 mW, CNI) was gated by an acousto- optic modulator and guided to the microscope frame by a single-mode fiber. A $60 \times$ oil objective was used to focus the laser beam to the samples and to collect the fluorescence. The fluorescence was filtered by a $650 \mathrm{~nm}$ long-pass filter and then measured by avalanche photodiodes (APDs; SPCM-AQRH15-FC, Excelitas). The fiber cores of the APDs also acted as the pinhole for the confocal imaging. A 3-axis piezo stage was used to scan the sample. A microwave from a signal generator (R\&S SMIQ03B) was gated by a radiofrequency switch, amplified (ZHL-16W-43+, Mini-Circuits), and then fed to a printed circuit board that held the samples. The pulse generator for pulse control and the time tagger for data acquisition were from Swabian Instruments. We used the one-step e-beam evaporation process to fabricate an $\Omega$-shaped antenna (100 nm/500 nm Cr/Au) on the cover glass with a designed metal mask. The antenna with a radius of $\sim 80 \mu \mathrm{m}$ was used to deliver microwave pulses to the NV centers.

The external magnetic field was generated by an electromagnet driven by a precise current source (TED4015, Thorlabs). To minimize the transverse magnetic field in our system, we first set the magnetic field at $\sim 4 \mathrm{mT}$ and finely tuned the orientation of the electromagnet to align the magnetic field along the NV axis as much as possible. This was achieved by minimizing the ground-state splitting of an NV center spin coupled to a first-shell ${ }^{13} \mathrm{C}$ nuclear spin (NV2$\mathrm{C} 13$ ), such that the splitting was less than the half width of the spin resonances [39]. Once the magnetic field was aligned, we kept the orientation of the magnetic field and decreased the current of the TED4015 to reduce the magnetic field. For all fields in the measurement, the ground-state spin splitting was negligible, which meant the transverse magnetic field in our system was $<0.1 \mathrm{mT}$. Such a small transverse magnetic field had negligible effects on our measurements. The magnetic field along the $z$ axis was calibrated by measuring the ODMR splitting of an NV center with a first-shell ${ }^{13} \mathrm{C}(\mathrm{NV} 2-\mathrm{C} 13)$ at different currents, which showed a linear dependence. From the linear curve fitting, we obtained the magnetic field strength at a given current. Note that the earth magnetic field along the $z$ axis was compensated.

NV central spin can be optically initialized to $m_{s}=0$ by optical excitation (using, e.g., $532 \mathrm{~nm}$ laser), manipulated by microwave pulses, and read out through spin-dependent photoluminescence. In the pulsed ODMR measurement, the photoluminescence was collected while the frequency of the microwave was swept. At each frequency, the spin state was firstly polarized to $m_{s}=0$ state, then flipped to $m_{s}=1$ by a $\pi$ pulse, and finally read out via spin-dependent photoluminescence under the excitation by the same green laser. When the microwave frequency was resonant with the energy level, there was a dip in the photoluminescence. For Ramsey measurement in the experiment, the spin state was first polarized to $m_{s}=0$ state, rotated to the superposition state by a $\frac{\pi}{2}$ pulse, then evolved for a free time $\tau$, and read out after another $\frac{\pi}{2}$ pulse. For spin echo measurement, an additional $\pi$ pulse was applied in the middle of the evolution time $\tau$ to inverse the spin dephasing. For $T_{1}$ relaxometry, the spin state was first prepared to $m_{s}=0$ or $m_{s}=+1 /-1$ state, then relaxed for a time $\tau$, and finally read out.

In our experiment, NV1 and NV2-C13 had the same orientation, which was confirmed by their nearly identical ODMR 

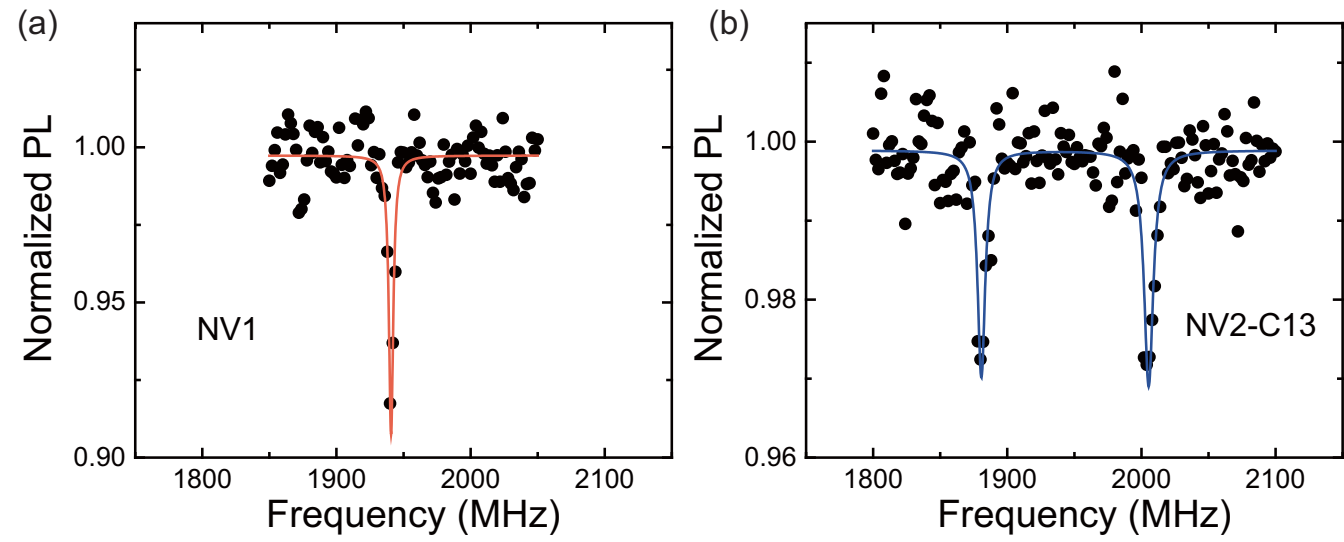

FIG. 5. Optically detected magnetic resonance (ODMR) spectra of NV1 and NV2-C13 under $B=33.2 \mathrm{mT}$.

splitting under $B=33.2 \mathrm{mT}$ as illustrated in Fig. 5. Figure 6 shows the ODMR spectra of NV1 and NV2-C13 under various weak magnetic fields.

\section{ACKNOWLEDGMENTS}

This paper was supported by Hong Kong Research Grants Council Collaborative Research Fund Project C4007-19G, Hong Kong Research Grant Council-French ANR Joint Scheme Project A-CUHK404/18, and The Chinese University of Hong Kong Group Research Grants. The authors acknowledge discussions with $\mathrm{V}$. Jacques on the effects of noises on shallow NV centers.

\section{APPENDIX A: HAMILTONIAN OF THE SYSTEM}

1. Hamiltonian of $\mathrm{NV}$ electron spin and ${ }^{15} \mathrm{~N}$ nuclear spin

The Hamiltonian of NV center is

$$
\begin{aligned}
H= & \left(D_{\mathrm{gs}}+\Pi_{z}\right) S_{z}^{2}+\Pi_{x}\left(S_{y} S_{y}-S_{x} S_{x}\right)+\Pi_{y}\left(S_{x} S_{y}+S_{y} S_{x}\right) \\
& +\gamma_{\mathrm{e}} \mathbf{B} \cdot \mathbf{S}+\mathbf{S} \cdot \mathbb{A}_{\mathrm{N}} \cdot \mathbf{I}_{\mathrm{N}},
\end{aligned}
$$

where $\mathbb{A}_{N}$ is the hyperfine tensor between the central electron spin and the ${ }^{15} \mathrm{~N}$ nuclear spin, which has only diagonal elements [38] as $A_{\mathrm{N}, x x}=A_{\mathrm{N}, y y}=3.65 \mathrm{MHz}$, $A_{\mathrm{N}, z z}=3.15 \mathrm{MHz}$. We set the $x y$ axes such that $\Pi_{y}=0$. Near zero field, as the transverse magnetic field $\gamma_{\mathrm{e}} B_{\perp} \ll D_{\mathrm{gs}}$, we only consider the magnetic field along $z$ axis. For the same reason, we also neglect the transverse hyperfine coupling $A_{\mathrm{N}, x x}$ and $A_{\mathrm{N}, y y}$. The Hamiltonian of the system becomes

$H \approx\left(D_{\mathrm{gs}}+\Pi_{z}\right) S_{z}^{2}+\Pi_{x}\left(S_{y} S_{y}-S_{x} S_{x}\right)+\gamma_{\mathrm{e}} B_{z} S_{z}+A_{\mathrm{N}, z z} S_{z} I_{\mathrm{N}, z}$.

The eigenvalues of the Hamiltonian are

$$
f_{m_{s}, \mu_{\mathrm{N}}}=D_{\mathrm{gs}}+\Pi_{z}+m_{s} \sqrt{\left(\gamma_{\mathrm{e}} B+\mu_{\mathrm{N}} A_{\mathrm{N}}\right)^{2}+\Pi_{x}^{2}} .
$$

Here, $A_{\mathrm{N}}=A_{\mathrm{N}, z z}=3.15 \mathrm{MHz}$ is the hyperfine interaction along the $z$ axis.

\section{Hamiltonian of $\mathrm{NV}$ electron spin and ${ }^{15} \mathrm{~N}$ nuclear spin and ${ }^{13} \mathrm{C}$ nuclear spins}

Then we consider the coupling to a first-shell ${ }^{13} \mathrm{C}$ nuclear spin $\mathbf{I}_{\mathbf{C}}$. The Hamiltonian is

$$
\begin{aligned}
H= & \left(D_{\mathrm{gs}}+\Pi_{z}\right) S_{z}^{2}+\Pi_{x}\left(S_{y} S_{y}-S_{x} S_{x}\right)+\gamma_{\mathrm{e}} B_{z} S_{z}+\mathbf{S} \cdot \mathbb{A}_{\mathbf{N}} \cdot \mathbf{I}_{\mathrm{N}} \\
& +\mathbf{S} \cdot \mathbb{A}_{\mathrm{C}} \cdot \mathbf{I}_{\mathrm{C}} .
\end{aligned}
$$

The hyperfine tensor $\mathbb{A}_{C}$ for a first-shell ${ }^{13} \mathrm{C}$ spin is $[39,53]$

$$
\mathbb{A}_{\mathrm{C}}=\left[\begin{array}{ccc}
A_{\mathrm{C}, x x^{\prime}} & 0 & A_{\mathrm{C}, x z} \\
0 & A_{\mathrm{C}, y y^{\prime}} & 0 \\
A_{\mathrm{C}, z x^{\prime}} & 0 & A_{\mathrm{C}, z z}
\end{array}\right],
$$
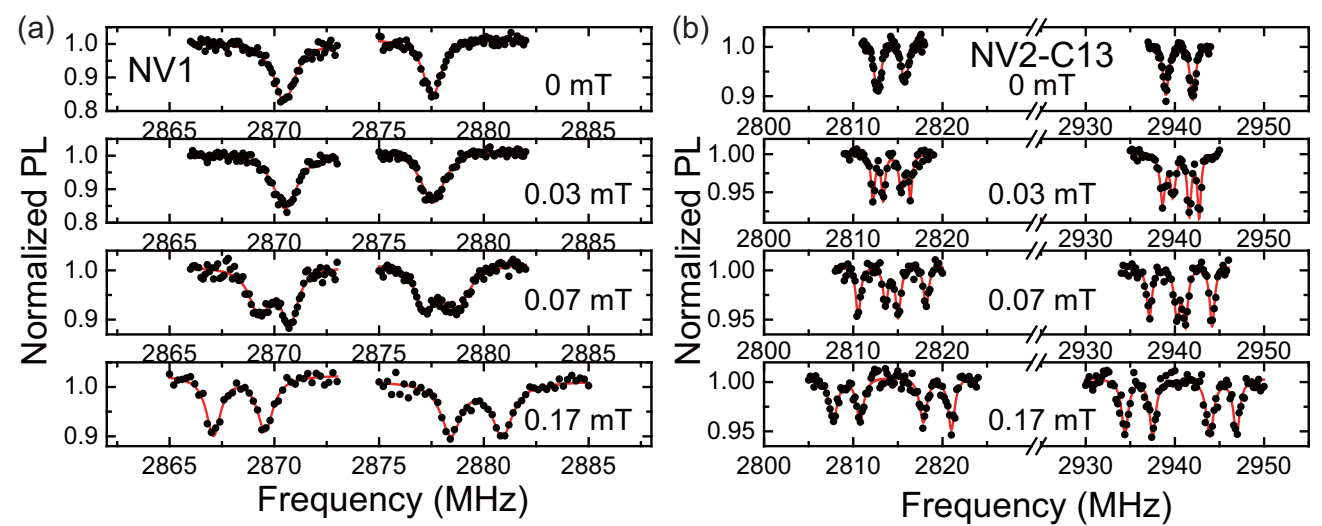

FIG. 6. Optically detected magnetic resonance (ODMR) spectra of NV1 and NV2-C13 under various weak magnetic fields. 
with $A_{\mathrm{C}, x^{\prime} x^{\prime}}=189.3 \mathrm{MHz}, A_{\mathrm{C}, y^{\prime} y^{\prime}}=128.4 \mathrm{MHz}, A_{\mathrm{C}, z z}=$ 128.0 MHz, and $A_{\mathrm{C}, x^{\prime} z / z x^{\prime}}=24.1 \mathrm{MHz}$ [40]. Note that the $x^{\prime}$ axis (set in the plane of the NV axis and the displacement of the ${ }^{13} \mathrm{C}$ ) is not necessarily the same as the $x$ axis defined by the strain.

Under the secular approximation (with the terms including $S_{x / y}$ dropped), the Hamiltonian in Eq. (1) in the main text is obtained as

$$
\begin{aligned}
H= & \left(D_{\mathrm{gs}}+\Pi_{z}\right) S_{z}^{2}+\Pi_{x}\left(S_{y} S_{y}-S_{x} S_{x}\right)+\gamma_{\mathrm{e}} B_{z} S_{z}+S_{z} \mathbf{A}_{\mathrm{N}} \cdot \mathbf{I}_{\mathrm{N}} \\
& +S_{z} \mathbf{A}_{\mathrm{C}} \cdot \mathbf{I}_{\mathrm{C}},
\end{aligned}
$$

where $\mathbf{A}_{\mathrm{N}}=A_{\mathrm{N}, z z} \mathbf{e}_{z}$ and $\mathbf{A}_{\mathrm{C}}=A_{\mathrm{C}, z x^{\prime}} \mathbf{e}_{x^{\prime}}+A_{\mathrm{C}, z z} \mathbf{e}_{z}$ are the hyperfine coupling vectors for the nitrogen and ${ }^{13} \mathrm{C}$ spins when the NV center spin is along the $z$ axis. The eigenenergies of the Hamiltonian are

$f_{m_{s}, \mu_{\mathrm{N}}, \mu_{\mathrm{C}}}=D_{\mathrm{gs}}+\Pi_{z}+m_{s} \sqrt{\left(\gamma_{\mathrm{e}} B+\mu_{\mathrm{N}} A_{\mathrm{N}}+\mu_{\mathrm{C}} A_{\mathrm{C}}\right)^{2}+\Pi_{x}^{2}}$,

with $A_{\mathrm{C}}=\sqrt{A_{\mathrm{C}, z x^{\prime}}^{2}+A_{\mathrm{C}, z z}^{2}} \approx 130 \mathrm{MHz}$.

\section{APPENDIX B: DECAY TIME OF RAMSEY INTERFERENCE}

The coherence time $T_{2}^{*}$ near the clock transition was measured by Ramsey interference. We denote the electrical fluctuations along different axes as $\epsilon_{x / y / z}$ and magnetic noise along the $z$ axis as $b$. Considering the transition between the states with $m_{\mathrm{s}}=0$ and $m_{\mathrm{s}}=+1$, e.g., the resonance frequency is

$$
\omega_{\mu}=D_{\mathrm{gs}}+\Pi_{z}+\epsilon_{z}+\sqrt{\left(B_{\mu}+b\right)^{2}+\left(\Pi_{x}+\epsilon_{x}\right)^{2}+\epsilon_{y}^{2}},
$$

where $B_{\mu}=\gamma_{\mathrm{e}} B_{z}+\mu_{\mathrm{N}} A_{\mathrm{N}} / 2$ for NV1 and $B_{\mu}=\gamma_{\mathrm{e}} B_{z}+$ $\mu_{\mathrm{N}} A_{\mathrm{N}} / 2+\mu_{\mathrm{C}} A_{\mathrm{C}} / 2$ for NV2-C13. The Ramsey coherence of this transition is

$$
L(t)=\left\langle\exp \left(-i \omega_{\mu} t\right)\right\rangle,
$$

which is averaged over the noises. We assume that both the magnetic noise and the electrical noise are Gaussian, with respective widths $\sigma_{b}$ and $\sigma_{x / y / z}$. Since usually the strength of the electric field coupling to the $z$ axis is much smaller than that coupling to the $x$ or $y$ axis $\left(\epsilon_{z} \ll \epsilon_{x / y}\right)$ [7], in the following, we drop $\epsilon_{z}$.
By Taylor expansion up to the leading order of the noises (which are much smaller than $\Pi_{x}$ ), we get

$$
\omega_{\mu} \approx \Delta_{\mu}+\frac{B_{\mu} b}{\Delta_{\mu}}+\frac{\epsilon_{x} \Pi_{x}}{\Delta_{\mu}},
$$

with $\Delta_{\mu}=\sqrt{B_{\mu}+\Pi_{x}^{2}}$. After Gaussian integration over the noise distribution, we obtain

$$
L(t) \approx \exp \left(-\frac{\sigma_{x}^{2} \Pi_{x}^{2}+\sigma_{b}^{2} B_{\mu}^{2}}{2 \Delta_{\mu}^{2}} t^{2}\right) \equiv \exp \left[-\left(\frac{t}{T_{2}^{*}}\right)^{2}\right]
$$

For fields far away from the LAC, $B_{\mu} \gg \Pi_{x}$ and $\Delta_{\mu} \approx B_{\mu}$, the electrical noise can be neglected, and therefore, $L(t) \propto$ $\exp \left(-\frac{\sigma_{b}^{2} t^{2}}{2}\right)$, with the coherence time independent of the magnetic field. Near the LAC (NV1 at zero or low field or NV2-C13 at a field $\left.B \approx \pm \frac{A_{\mathrm{C}}}{2 \gamma_{\mathrm{e}}} \approx \pm 2.3 \mathrm{mT}\right), B_{\mu} \sim \Pi_{x}$, and the electrical noise becomes important.

We fit the measured Ramsey signal using Eq. (B4) to obtain $T_{2}^{*}$ under different magnetic fields. The Ramsey signals under different magnetic fields for NV1 and NV2-C13 are shown in Fig. 7. For NV2-C13, the Ramsey signals are obtained by the difference between Ramsey interference of initialization state $m_{s}=0$ and that of $m_{s}=-1$ to eliminate the fluctuations of the system. Different methods do not affect the fitted results of $T_{2}^{*}$.

\section{APPENDIX C: PULSE SEQUENCES FOR RELAXOMETRY}

We used an NV center coupled to a first-shell ${ }^{13} \mathrm{C}$ nuclear spin to measure the noise spectra of single MNPs. The coppernickel MNPs used here are ferromagnetic nanoparticles with sizes $\sim 100-200 \mathrm{~nm}$. Detailed information about the nanoparticles can be found in Ref. [41]. The magnetic particles are in the ferromagnetic state, which was confirmed by the measured splitting of the spin resonance from its net magnetization and is consistent with their sizes (which are larger than typical superparamagnetic MNPs).

The relaxation dynamics of an NV center spin strongly coupled to a ${ }^{13} \mathrm{C}$ nuclear spin is illustrated in Fig. 8(a). Since the relaxation time of the nuclear spin is much longer than that of the electron spin, the nuclear spin is conserved during the relaxation of the electron spin, resulting in two independent processes [left and right panels in Fig. 8(a)]. The relaxation dynamics is reduced to a three-level system with the rate equation as

$$
\frac{d}{d t}\left(\begin{array}{c}
P_{0, \mp 1 / 2} \\
P_{+1, \mp 1 / 2} \\
P_{-1, \mp 1 / 2}
\end{array}\right)=\left(\begin{array}{ccc}
-\Gamma_{1 / 3}-\Gamma_{2 / 4} & \Gamma_{1 / 3} & \Gamma_{2 / 4} \\
\Gamma_{1 / 3} & -\Gamma_{1 / 3}-\gamma_{\alpha / \beta} & \gamma_{\alpha / \beta} \\
\Gamma_{2 / 4} & \gamma_{\alpha / \beta} & -\Gamma_{2 / 4}-\gamma_{\alpha / \beta}
\end{array}\right)\left(\begin{array}{c}
P_{0, \mp 1 / 2} \\
P_{+1, \mp 1 / 2} \\
P_{-1, \mp 1 / 2}
\end{array}\right),
$$

where $P_{m_{s}, \mu_{C}}$ is the population of the corresponding spin state, and $\Gamma_{i}$ and $\gamma_{\alpha / \beta}$ are the relaxations rates for different transitions shown in Fig. 8(a). When there is an MNP in the proximity, the main mechanism of the spin relaxation is the Johnson and magnetic noise from the nanoparticle, which have little effect on the transition between $\left|m_{s}=+1\right\rangle$ and $\left|m_{s}=-1\right\rangle$, characterized by the rates $\gamma_{\alpha / \beta}$.

Figure 8(b) shows the pulse sequences for $T_{1}$ measurement. To exclude the effects of fluorescence decay due to spinindependent processes (such as the charge state jumps), we used the difference between the signals with and without a $\pi$ pulse between the states $\left|0, \mu_{\mathrm{C}}\right\rangle$ and $\left| \pm 1, \mu_{\mathrm{C}}\right\rangle$ right before readout. We first polarized the spin state to the states $\left|m_{s}=0, \mu_{\mathrm{C}}= \pm \frac{1}{2}\right\rangle$ and let the system relax for a time $t$. At the end of the sequence, we measured the spin-dependent fluorescence either directly or after applying a $\pi$ pulse on the transition. The signals are denoted as $s_{0}$ (without a $\pi$ pulse) and $s_{i}$ (with a $\pi$ pulse applied to the 

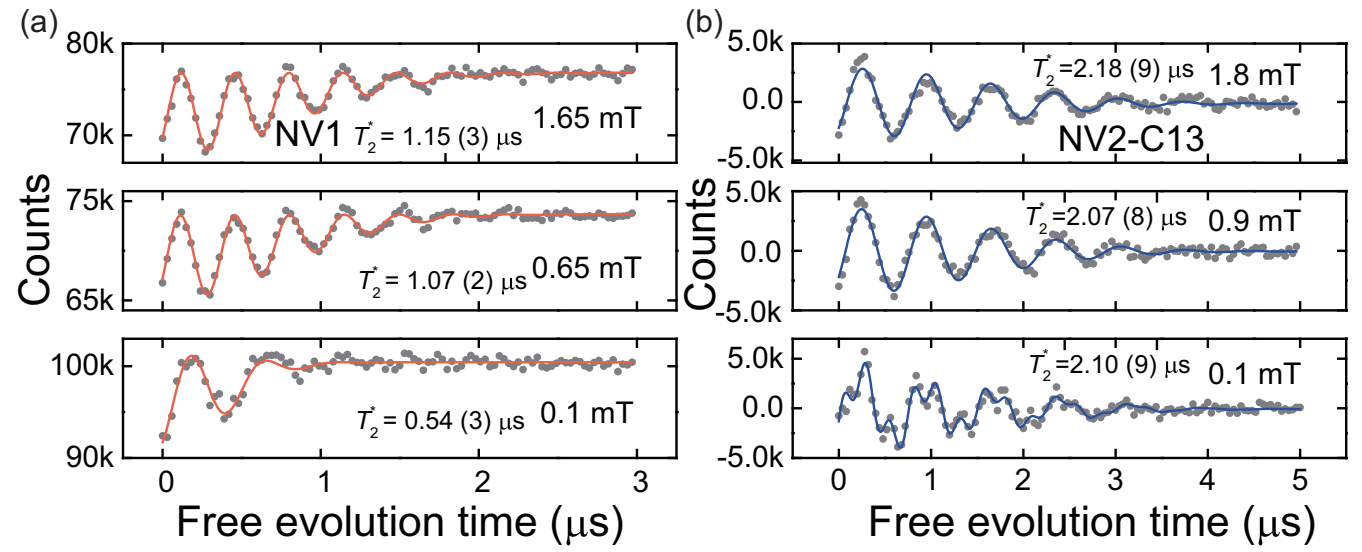

FIG. 7. Ramsey interference signals of NV1 and NV2-13C under different magnetic fields.

$i$ th transition with the relaxation rate $\left.\Gamma_{i}\right)$. The signals are related to the populations in different states by

$$
\begin{aligned}
& s_{0}=A\left(P_{0,-1 / 2}+P_{0,+1 / 2}\right)+B\left(P_{-1,-1 / 2}+P_{+1,-1 / 2}+P_{-1,+1 / 2}+P_{+1,+1 / 2}\right)+C, \\
& s_{1}=A\left(P_{+1,-1 / 2}+P_{0,+1 / 2}\right)+B\left(P_{-1,-1 / 2}+P_{0,-1 / 2}+P_{-1,+1 / 2}+P_{+1,+1 / 2}\right)+C, \\
& s_{2}=A\left(P_{-1,-1 / 2}+P_{0,+1 / 2}\right)+B\left(P_{0,-1 / 2}+P_{+1,-1 / 2}+P_{-1,+1 / 2}+P_{+1,+1 / 2}\right)+C, \\
& s_{3}=A\left(P_{0,-1 / 2}+P_{+1,+1 / 2}\right)+B\left(P_{-1,-1 / 2}+P_{+1,-1 / 2}+P_{-1,+1 / 2}+P_{0,+1 / 2}\right)+C, \\
& s_{4}=A\left(P_{0,-1 / 2}+P_{-1,+1 / 2}\right)+B\left(P_{-1,-1 / 2}+P_{+1,-1 / 2}+P_{0,+1 / 2}+P_{+1,+1 / 2}\right)+C,
\end{aligned}
$$

where $A$ and $B$ are the photon counts for the $\left|m_{s}=0\right\rangle$ and $\left|m_{s}= \pm 1\right\rangle$ states, respectively, and $C$ is for the background photon counts. With the relation $P_{0, \mu_{\mathrm{C}}}+P_{+1, \mu_{\mathrm{C}}}+P_{-1, \mu_{\mathrm{C}}}=\frac{1}{2}$, we obtain the populations of each spin state from the photon counts as

$$
\begin{aligned}
P_{0, \mp 1 / 2} & =\frac{1}{6}+\frac{2 s_{0}-\left(s_{1 / 3}+s_{2 / 4}\right)}{3(A-B)}, \\
P_{+1, \mp 1 / 2} & =\frac{1}{6}+\frac{2 s_{1 / 3}-\left(s_{0}+s_{2 / 4}\right)}{3(A-B)}, \\
P_{-1, \mp 1 / 2} & =\frac{1}{6}+\frac{2 s_{2 / 4}-\left(s_{0}+s_{1 / 3}\right)}{3(A-B)} .
\end{aligned}
$$

For signals more sensitive to the transition rates $\gamma_{\alpha / \beta}$, we measured the double-quantum (DQ) relaxation [27]. Figure 8(c) is the sequence for measuring the DQ relaxation between $\left|m_{s}=+1 ; \mu_{\mathrm{C}}\right\rangle$ and $\left|m_{s}=-1 ; \mu_{\mathrm{C}}\right\rangle$. We first prepared the states to $\left|m_{s}=+1 ; \mu_{\mathrm{C}}\right\rangle$ and let the system evolve a time $t$. At the end of the sequence, an additional $\pi$ pulse was applied on the transition $\left|m_{s}=0 ; \mu_{\mathrm{C}}\right\rangle \leftrightarrow\left|m_{s}=+1 ; \mu_{\mathrm{C}}\right\rangle$ or $\left|m_{s}=0 ; \mu_{\mathrm{C}}\right\rangle \leftrightarrow\left|m_{s}=-1 ; \mu_{\mathrm{C}}\right\rangle$ before readout. The signal $s_{i j}$ is for the two $\pi$ pulses applied for the $i$ th and $j$ th transitions. The measured signals are related to the populations in different states as

$$
\begin{aligned}
& s_{11}=A\left(P_{+1,-1 / 2}^{\prime}+P_{0,+1 / 2}^{\prime}\right)+B\left(P_{-1,-1 / 2}^{\prime}+P_{0,-1 / 2}^{\prime}+P_{-1,+1 / 2}^{\prime}+P_{+1,+1 / 2}^{\prime}\right)+C, \\
& s_{12}=A\left(P_{-1,-1 / 2}^{\prime}+P_{0,+1 / 2}^{\prime}\right)+B\left(P_{0,-1 / 2}^{\prime}+P_{+1,-1 / 2}^{\prime}+P_{-1,+1 / 2}^{\prime}+P_{+1,+1 / 2}^{\prime}\right)+C, \\
& s_{33}=A\left(P_{0,-1 / 2}^{\prime}+P_{+1,+1 / 2}^{\prime}\right)+B\left(P_{-1,-1 / 2}^{\prime}+P_{+1,-1 / 2}^{\prime}+P_{-1,+1 / 2}^{\prime}+P_{0,+1 / 2}^{\prime}\right)+C, \\
& s_{34}=A\left(P_{0,-1 / 2}^{\prime}+P^{\prime}{ }_{-1,+1 / 2}\right)+B\left(P_{-1,-1 / 2}^{\prime}+P^{\prime}{ }_{+1,-1 / 2}+P_{0,+1 / 2}^{\prime}+P_{+1,+1 / 2}^{\prime}\right)+C .
\end{aligned}
$$

Here, $P_{m_{s}, \mu_{C}}$ differ from $P_{m_{s}, \mu_{C}}$ for their different initial conditions. The DQ relaxations between $\left|m_{s}=+1 ; \mu_{\mathrm{C}}\right\rangle$ and $\mid m_{s}=-1$; $\left.\mu_{\mathrm{C}}\right\rangle$ are

$$
\begin{aligned}
& S_{\mathrm{DQ},-1 / 2}=P_{+1,-1 / 2}^{\prime}-P_{-1,-1 / 2}^{\prime}=\frac{s_{11}-s_{12}}{A-B}, \\
& S_{\mathrm{DQ},+1 / 2}=P_{+1,+1 / 2}^{\prime}-P_{-1,+1 / 2}^{\prime}=\frac{s_{33}-s_{34}}{A-B} .
\end{aligned}
$$

The measured populations and DQ relaxation signals of NV3-13C with an MNP nearby are shown in Fig. 9. The upper panels of Figs. 9(a) and 9(b) show the populations for $\left|m_{s}=0, \pm 1, \mu_{\mathrm{C}}\right\rangle$ obtained with Eq. (C3), and the lower panels show the DQ relaxations between $\left|m_{s}=+1, \mu_{\mathrm{C}}\right\rangle$ and $\left|m_{s}=-1, \mu_{\mathrm{C}}\right\rangle$ obtained with Eq. (C5). We fit the populations in $\left|m_{s}, \mu_{\mathrm{C}}=-\frac{1}{2}\right\rangle$ and the DQ signal for $\mu_{\mathrm{C}}=-\frac{1}{2}$ [curves in Fig. 9(a)] by solving the rate equations in Eq. (C1) with their respective initial states, and obtain the best fitted relaxation rates $\Gamma_{1}=10.0(5) \mathrm{ms}^{-1}, \Gamma_{2}=5.0(4) \mathrm{ms}^{-1}$, and $\gamma_{\alpha}=0.5(2) \mathrm{ms}^{-1}$. Similarly, we fit the curves 
(a)

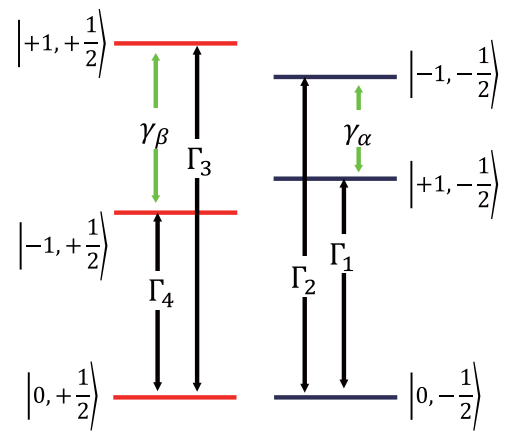

(b)

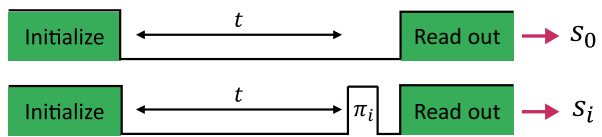

(c)

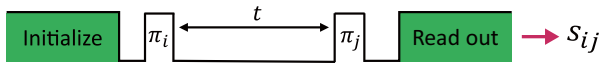

FIG. 8. Relaxation dynamics and pulse sequences for $T_{1}$ measurement. (a) Relaxation rates between different states. The transition rate $\left(\gamma_{\alpha / \beta}\right)$ between $\left|m_{s}=+1\right\rangle$ and $\left|m_{s}=-1\right\rangle$ is much smaller than $\Gamma_{i}$ when the noise is mainly from the magnetic nanoparticle. (b) Pulse sequences to measure the $T_{1}$ time. (c) Pulse sequences to measure double-quantum relaxations.

in Fig. 9(b) and get the relaxation rates $\Gamma_{3}=6.0(4) \mathrm{ms}^{-1}, \Gamma_{4}=8.9(5) \mathrm{ms}^{-1}$, and $\gamma_{\beta}=0.5(2) \mathrm{ms}^{-1}$. The measured relaxation rates $\Gamma_{i}$ increase dramatically as compared with the relaxation rate of NV3-C13 [0.15(1) ms $\left.{ }^{-1}\right]$ before inducing the MNP, which indicates that the noises are mainly from the nearby MNP. The relaxation rates $\Gamma_{1}$ and $\Gamma_{4}$ for the transitions with lower energies are significantly larger than $\Gamma_{2}$ and $\Gamma_{3}$ for the transitions with higher energies, and the transitions with similar frequencies have similar relaxation rates. These facts suggest that the noises from the MNP have a subgigahertz spectral width. The DQ relaxation rates are nearly the same $\left(\gamma_{\alpha}=\gamma_{\beta}\right)$ and are much smaller than the single-quantum relaxation rates $\Gamma_{i}$, which is consistent with the results in Ref. [19].
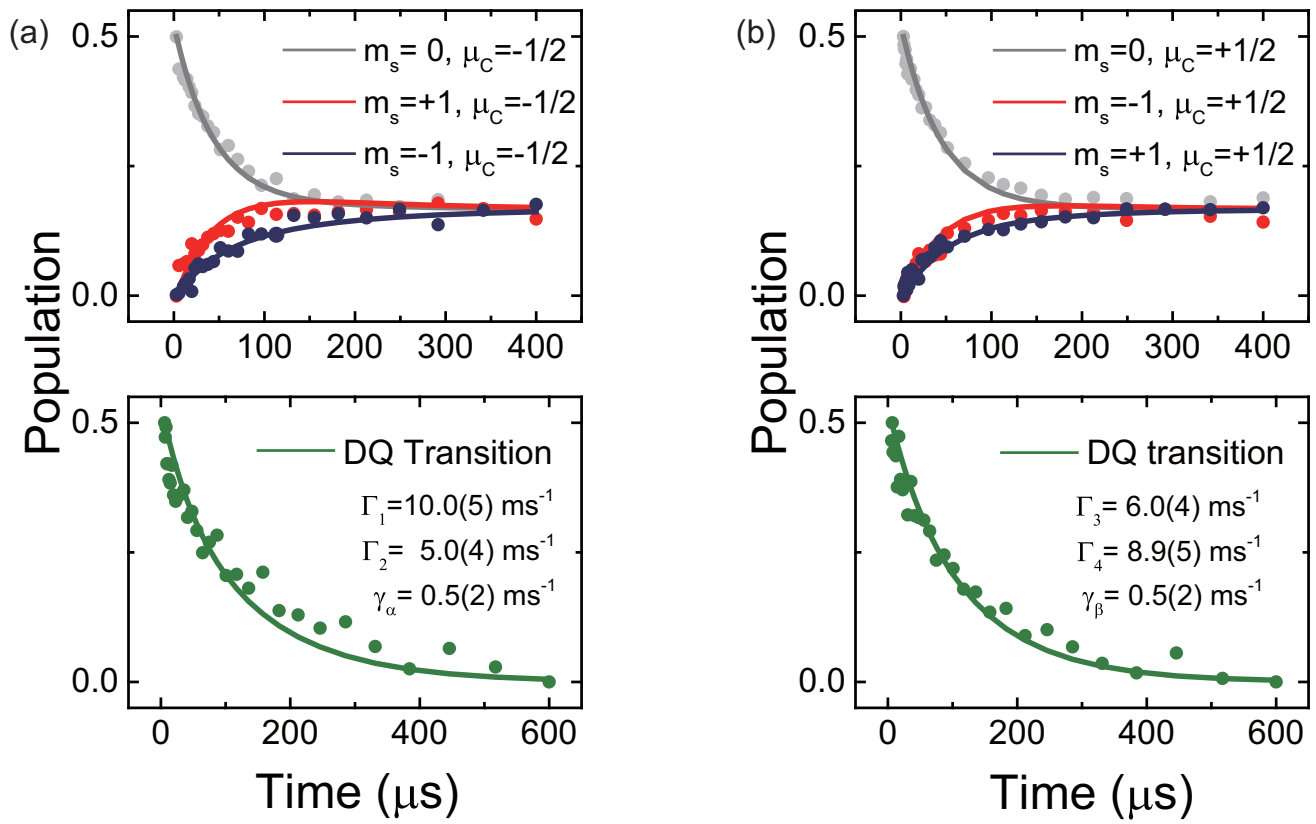

FIG. 9. Populations of the spin states and the double-quantum (DQ) signals between $\left|m_{s}=+1 ; \mu_{C}\right\rangle$ and $\left|m_{s}=-1 ; \mu_{C}\right\rangle$. (a) Upper: populations of the states $\left|m_{s} ; \mu_{C}=-\frac{1}{2}\right\rangle$; lower: DQ relaxation between $\left|m_{s}=+1, \mu_{C}=-\frac{1}{2}\right\rangle$ and $\left|m_{s}=-1, \mu_{C}=-\frac{1}{2}\right\rangle$. (b) Upper: populations of the states $\left|m_{s} ; \mu_{C}=+\frac{1}{2}\right\rangle$; lower: DQ relaxation between $\left|m_{s}=+1, \mu_{C}=+\frac{1}{2}\right\rangle$ and $\left|m_{s}=-1, \mu_{C}=+\frac{1}{2}\right\rangle$. The dots are measured spin populations and DQ relaxations, and the curves are fitting results from Eq. (C1).

[1] A. Gruber, A. Dräbenstedt, C. Tietz, L. Fleury, J. Wrachtrup, and C. Von Borczyskowski, Scanning confocal optical microscopy and magnetic resonance on single defect centers, Science 276, 2012 (1997).
[2] F. Casola, T. van der Sar, and A. Yacoby, Probing condensed matter physics with magnetometry based on nitrogenvacancy centres in diamond, Nat. Rev. Mater. 3, 17088 (2018). 
[3] C. L. Degen, F. Reinhard, and P. Cappellaro, Quantum sensing, Rev. Mod. Phys. 89, 035002 (2017).

[4] J. R. Maze, P. L. Stanwix, J. S. Hodges, S. Hong, J. M. Taylor, P. Cappellaro, L. Jiang, M. V. G. Dutt, E. Togan, A. S. Zibrov, A. Yacoby, R. L. Walsworth, and M. D. Lukin, Nanoscale magnetic sensing with an individual electronic spin in diamond, Nature 455, 644 (2008).

[5] G. Balasubramanian, I. Y. Chan, R. Kolesov, M. Al-Hmoud, J. Tisler, C. Shin, C. Kim, A. Wojcik, P. R. Hemmer, A. Krueger, T. Hanke, A. Leitenstorfer, R. Bratschitsch, F. Jelezko, and J. Wrachtrup, Nanoscale imaging magnetometry with diamond spins under ambient conditions, Nature 455, 648 (2008).

[6] J. M. Taylor, P. Cappellaro, L. Childress, L. Jiang, D. Budker, P. R. Hemmer, A. Yacoby, R. Walsworth, and M. D. Lukin, Highsensitivity diamond magnetometer with nanoscale resolution, Nat. Phys. 4, 810 (2008).

[7] F. Dolde, H. Fedder, M. W. Doherty, T. Nöbauer, F. Rempp, G. Balasubramanian, T. Wolf, F. Reinhard, L. C. L. Hollenberg, F. Jelezko, and J. Wrachtrup, Electric-field sensing using single diamond spins, Nat. Phys. 7, 459 (2011).

[8] V. M. Acosta, E. Bauch, M. P. Ledbetter, A. Waxman, L. S. Bouchard, and D. Budker, Temperature Dependence of the Nitrogen-Vacancy Magnetic Resonance in Diamond, Phys. Rev. Lett. 104, 070801 (2010).

[9] P. Neumann, I. Jakobi, F. Dolde, C. Burk, R. Reuter, G. Waldherr, J. Honert, T. Wolf, A. Brunner, J. H. Shim, D. Suter, H. Sumiya, J. Isoya, and J. Wrachtrup, High-precision nanoscale temperature sensing using single defects in diamond, Nano Lett. 13, 2738 (2013).

[10] G. Kucsko, P. C. Maurer, N. Y. Yao, M. Kubo, H. J. Noh, P. K. Lo, H. Park, and M. D. Lukin, Nanometre-scale thermometry in a living cell, Nature 500, 54 (2013).

[11] D. M. Toyli, C. F. de las Casas, D. J. Christle, V. V. Dobrovitski, and D. D. Awschalom, Fluorescence thermometry enhanced by the quantum coherence of single spins in diamond, Proc. Natl. Acad. Sci. USA 110, 8417 (2013).

[12] M. W. Doherty, V. V. Struzhkin, D. A. Simpson, L. P. McGuinness, Y. Meng, A. Stacey, T. J. Karle, R. J. Hemley, N. B. Manson, L. C. L. Hollenberg, and S. Prawer, Electronic Properties and Metrology Applications of the Diamond $\mathrm{NV}^{-}$ Center under Pressure, Phys. Rev. Lett. 112, 047601 (2014).

[13] K. Xia, C.-F. Liu, W.-H. Leong, M.-H. Kwok, Z.-Y. Yang, X. Feng, R.-B. Liu, and Q. Li, Nanometer-precision nonlocal deformation reconstruction using nanodiamond sensing, Nat. Commun. 10, 3259 (2019).

[14] M. H. Abobeih, J. Cramer, M. A. Bakker, N. Kalb, M. Markham, D. J. Twitchen, and T. H. Taminiau, One-second coherence for a single electron spin coupled to a multi-qubit nuclear-spin environment, Nat. Commun. 9, 2552 (2018).

[15] M. Pelliccione, A. Jenkins, P. Ovartchaiyapong, C. Reetz, E. Emmanouilidou, N. Ni, and A. C. Bleszynski Jayich, Scanned probe imaging of nanoscale magnetism at cryogenic temperatures with a single-spin quantum sensor, Nat. Nanotech. 11, 700 (2016).

[16] L. Thiel, D. Rohner, M. Ganzhorn, P. Appel, E. Neu, B. Müller, R. Kleiner, D. Koelle, and P. Maletinsky, Quantitative nanoscale vortex imaging using a cryogenic quantum magnetometer, Nat. Nanotech. 11, 677 (2016).

[17] Y. Dovzhenko, F. Casola, S. Schlotter, T. X. Zhou, F. Büttner, R. L. Walsworth, G. S. D. Beach, and A. Yacoby, Magnetostatic twists in room-temperature skyrmions explored by nitrogenvacancy center spin texture reconstruction, Nat. Commun. 9, 2712 (2018).

[18] A. Jenkins, M. Pelliccione, G. Yu, X. Ma, X. Li, K. L. Wang, and A. C. B. Jayich, Single-spin sensing of domain-wall structure and dynamics in a thin-film skyrmion host, Phys. Rev. Materials 3, 083801 (2019).

[19] C. Du, T. van der Sar, T. X. Zhou, P. Upadhyaya, F. Casola, H. Zhang, M. C. Onbasli, C. A. Ross, R. L. Walsworth, Y. Tserkovnyak, and A. Yacoby, Control and local measurement of the spin chemical potential in a magnetic insulator, Science 357, 195 (2017).

[20] P. Andrich, C. F. de las Casas, X. Liu, H. L. Bretscher, J. R. Berman, F. J. Heremans, P. F. Nealey, and D. D. Awschalom, Long-range spin wave mediated control of defect qubits in nanodiamonds, npj Quantum Inf. 3, 28 (2017).

[21] I. Bertelli, J. J. Carmiggelt, T. Yu, B. G. Simon, C. C. Pothoven, G. E. W. Bauer, Y. M. Blanter, J. Aarts, and T. van der Sar, Magnetic resonance imaging of spin-wave transport and interference in a magnetic insulator, Sci. Adv. 6, eabd3556 (2020).

[22] I. Gross, W. Akhtar, V. Garcia, L. J. Martínez, S. Chouaieb, K. Garcia, C. Carrétéro, A. Barthélémy, P. Appel, P. Maletinsky, J.-V. Kim, J. Y. Chauleau, N. Jaouen, M. Viret, M. Bibes, S. Fusil, and V. Jacques, Real-space imaging of non-collinear antiferromagnetic order with a single-spin magnetometer, Nature 549, 252 (2017).

[23] P. Appel, B. J. Shields, T. Kosub, N. Hedrich, R. Hübner, J. Faßbender, D. Makarov, and P. Maletinsky, Nanomagnetism of magnetoelectric granular thin-film antiferromagnets, Nano Lett. 19, 1682 (2019).

[24] L. Thiel, Z. Wang, M. A. Tschudin, D. Rohner, I. GutiérrezLezama, N. Ubrig, M. Gibertini, E. Giannini, A. F. Morpurgo, and P. Maletinsky, Probing magnetism in 2D materials at the nanoscale with single-spin microscopy, Science 364, 973 (2019).

[25] B. A. Myers, A. Das, M. C. Dartiailh, K. Ohno, D. D Awschalom, and A. C. Bleszynski Jayich, Probing Surface Noise with Depth-Calibrated Spins in Diamond, Phys. Rev. Lett. 113, 027602 (2014).

[26] T. Rosskopf, A. Dussaux, K. Ohashi, M. Loretz, R. Schirhagl, H. Watanabe, S. Shikata, K. M. Itoh, and C. L. Degen, Investigation of Surface Magnetic Noise by Shallow Spins in Diamond, Phys. Rev. Lett. 112, 147602 (2014).

[27] B. A. Myers, A. Ariyaratne, and A. C. Bleszynski Jayich, Double-Quantum Spin-Relaxation Limits to Coherence of Near-Surface Nitrogen-Vacancy Centers, Phys. Rev. Lett. 118, 197201 (2017).

[28] S. Sangtawesin, B. L. Dwyer, S. Srinivasan, J. J. Allred, L. V. H. Rodgers, K. De Greve, A. Stacey, N. Dontschuk, K. M. O’Donnell, D. Hu, D. A. Evans, C. Jaye, D. A. Fischer, M. L. Markham, D. J. Twitchen, H. Park, M. D. Lukin, and N. P. de Leon, Origins of Diamond Surface Noise Probed by Correlating Single-Spin Measurements with Surface Spectroscopy, Phys. Rev. X 9, 031052 (2019).

[29] P. C. Hohenberg and B. I. Halperin, Theory of dynamic critical phenomena, Rev. Mod. Phys. 49, 435 (1977).

[30] A. P. Roberts, T. P. Almeida, N. S. Church, R. J. Harrison, D. Heslop, Y. Li, J. Li, A. R. Muxworthy, W. Williams, and X. Zhao, Resolving the origin of pseudo-single domain magnetic behavior, J. Geophys. Res. Solid Earth 122, 9534 (2017). 
[31] C. Jin, Z. Tao, K. Kang, K. Watanabe, T. Taniguchi, K. F. Mak, and J. Shan, Imaging and control of critical fluctuations in twodimensional magnets, Nat. Mater. 19, 1290 (2020).

[32] J. Zázvorka, F. Dittrich, Y. Ge, N. Kerber, K. Raab, T. Winkler, K. Litzius, M. Veis, P. Virnau, and M. Kläui, Skyrmion lattice phases in thin film multilayer, Adv. Funct. Mater. 30, 2004037 (2020).

[33] N. Kerber, M. Weißenhofer, K. Raab, K. Litzius, J. Zázvorka, U. Nowak, and M. Kläui, Anisotropic Skyrmion Diffusion Controlled by Magnetic-Field-Induced Symmetry Breaking, Phys. Rev. Applied 15, 044029 (2021).

[34] G. Wolfowicz, A. M. Tyryshkin, R. E. George, H. Riemann, N. V. Abrosimov, P. Becker, H.-J. Pohl, M. L. W. Thewalt, S. A. Lyon, and J. J. L. Morton, Atomic clock transitions in siliconbased spin qubits, Nat. Nanotech. 8, 561 (2013).

[35] M. Shiddiq, D. Komijani, Y. Duan, A. Gaita-Ariño, E. Coronado, and S. Hill, Enhancing coherence in molecular spin qubits via atomic clock transitions, Nature 531, 348 (2016).

[36] P. Jamonneau, M. Lesik, J. P. Tetienne, I. Alvizu, L. Mayer, A. Dréau, S. Kosen, J.-F. Roch, S. Pezzagna, J. Meijer, T. Teraji, Y. Kubo, P. Bertet, J. R. Maze, and V. Jacques, Competition between electric field and magnetic field noise in the decoherence of a single spin in diamond, Phys. Rev. B 93, 024305 (2016).

[37] S. A. Momenzadeh, R. J. Stöhr, F. F. de Oliveira, A. Brunner, A. Denisenko, S. Yang, F. Reinhard, and J. Wrachtrup, Nanoengineered diamond waveguide as a robust bright platform for nanomagnetometry using shallow nitrogen vacancy centers, Nano Lett. 15, 165 (2015).

[38] K. Ohno, F. Joseph Heremans, L. C. Bassett, B. A. Myers, D. M. Toyli, A. C. Bleszynski Jayich, C. J. Palmstrøm, and D. D. Awschalom, Engineering shallow spins in diamond with nitrogen delta-doping, Appl. Phys. Lett. 101, 082413 (2012).

[39] K. R. K. Rao and D. Suter, Characterization of hyperfine interaction between an NV electron spin and a first-shell ${ }^{13} \mathrm{C}$ nuclear spin in diamond, Phys. Rev. B 94, 060101(R) (2016).

[40] J. F. Barry, J. M. Schloss, E. Bauch, M. J. Turner, C. A. Hart, L. M. Pham, and R. L. Walsworth, Sensitivity optimization for NV-diamond magnetometry, Rev. Mod. Phys. 92, 015004 (2020).

[41] N. Wang, G.-Q. Liu, W.-H. Leong, H. Zeng, X. Feng, S.-H. Li, F. Dolde, H. Fedder, J. Wrachtrup, X.-D. Cui, S. Yang, Q. Li, and R.-B. Liu, Magnetic Criticality Enhanced Hybrid Nanodiamond Thermometer under Ambient Conditions, Phys. Rev. X 8, 011042 (2018).

[42] N. Zhao, J. Honert, B. Schmid, M. Klas, J. Isoya, M. Markham, D. Twitchen, F. Jelezko, R. B. Liu, H. Fedder, and J. Wrachtrup,
Sensing single remote nuclear spins, Nat. Nanotech. 7, 657 (2012).

[43] N. Zhao, S.-W. Ho, and R.-B. Liu, Decoherence and dynamical decoupling control of nitrogen vacancy center electron spins in nuclear spin baths, Phys. Rev. B 85, 115303 (2012).

[44] S. Kolkowitz, A. Safira, A. A. High, R. C. Devlin, S. Choi, Q. P. Unterreithmeier, D. Patterson, A. S. Zibrov, V. E. Manucharyan, H. Park, and M. D. Lukin, Probing Johnson noise and ballistic transport in normal metals with a single-spin qubit, Science 347, 1129 (2015).

[45] A. Ariyaratne, D. Bluvstein, B. A. Myers, and A. C. B. Jayich, Nanoscale electrical conductivity imaging using a nitrogenvacancy center in diamond, Nat. Commun. 9, 2406 (2018).

[46] H. Zheng, J. Xu, G. Z. Iwata, T. Lenz, J. Michl, B. Yavkin, K. Nakamura, H. Sumiya, T. Ohshima, J. Isoya, J. Wrachtrup, A. Wickenbrock, and D. Budker, Zero-Field Magnetometry Based on Nitrogen-Vacancy Ensembles in Diamond, Phys. Rev. Applied 11, 064068 (2019).

[47] T. Lenz, A. Wickenbrock, F. Jelezko, G. Balasubramanian, and D. Budker, Magnetic sensing at zero field with a single nitrogen-vacancy center, Quantum Sci. Technol. 6, 034006 (2021).

[48] J. Cerrillo, S. Oviedo Casado, and J. Prior, Low Field NanoNMR via Three-Level System Control, Phys. Rev. Lett. 126, 220402 (2021).

[49] P. J. Vetter, A. Marshall, G. T. Genov, T. F. Weiss, N. Striegler, E. F. Großmann, S. O. Casado, J. Cerrillo, J. Prior, P. Neumann, and F. Jelezko, Zero- and low-field nano-NMR with nitrogen vacancy centers, arXiv:2107.10537.

[50] B. Smeltzer, L. Childress, and A. Gali, ${ }^{13} \mathrm{C}$ hyperfine interactions in the nitrogen-vacancy centre in diamond, New J. Phys. 13, 025021 (2011).

[51] G. Balasubramanian, P. Neumann, D. Twitchen, M. Markham, R. Kolesov, N. Mizuochi, J. Isoya, J. Achard, J. Beck, J. Tissler, V. Jacques, P. R. Hemmer, F. Jelezko, and J. Wrachtrup, Ultralong spin coherence time in isotopically engineered diamond, Nat. Mater. 8, 383 (2009).

[52] N. Mizuochi, P. Neumann, F. Rempp, J. Beck, V. Jacques, P. Siyushev, K. Nakamura, D. J. Twitchen, H. Watanabe, S. Yamasaki, F. Jelezko, and J. Wrachtrup, Coherence of single spins coupled to a nuclear spin bath of varying density, Phys. Rev. B 80, 041201(R) (2009).

[53] G. A. Álvarez, C. O. Bretschneider, R. Fischer, P. London, H Kanda, S. Onoda, J. Isoya, D. Gershoni, and L. Frydman, Local and bulk ${ }^{13} \mathrm{C}$ hyperpolarization in nitrogen-vacancy-centred diamonds at variable fields and orientations, Nat. Commun. 6, 8456 (2015). 